\title{
Improving Link Spectrum Utilization in FlexGrid Optical Networks
}

\author{
Jaume Comellas and Gabriel Junyent
}

\begin{abstract}
Flexible grid optical networks emerge as an appealing solution for future high-speed optical transport, since they can provide superior flexibility and scalability, coping with the rapid growth of Internet traffic. This work introduces some simple strategies that help improving the spectrum usage in flexgrid optical networks, based on limiting the bandwidth flexibility of the requested connections. Link performance improvement is demonstrated by adapting fully elastic optical networks to multibitrate optical networks, where only some connection bandwidth values are allowed. With the aim of avoiding useless spectrum voids, the set of bandwidth values that connections can take is appropriately adjusted. It is analytically demonstrated that by limiting the possible connection sizes, some gain is obtained at the cost of losing some flexibility. Simulation results obtained at the link as well as at the whole network level assess the advantages anticipated by the theoretical study.
\end{abstract}

Index Terms - Elastic Optical Network; Grid-less Optical Network; Spectrum Management; Spectrum Allocation.

\section{INTRODUCTION}

$\mathbf{T}$ iggered by emerging services such as high-definition video distribution, the IP traffic volume has been exponentially increasing to date. Furthermore, day by day novel applications disclosed, as well as endless technology advances, guarantee that the traffic growth will not stop here. For example, new hardware advances such as multicore processing, virtualization and network storage will support a new generation of e-Science and cloud applications requesting data flows of $10 \mathrm{~Gb} / \mathrm{s}$ up to terabit level. The predictable consequence is that network operators will require a new generation of optical transport networks in the near future, so as to serve this huge and heterogeneous volume of traffic in a cost-effective and scalable manner [1]. In response to these large capacity and

Manuscript received February 24, 2015

The authors are with the Optical Communications Research Group, Universitat Politècnica de Catalunya, UPC Campus Nord D5, 08034 Barcelona, Spain. Tel: (+34) 93401 6441, (e-mail: comellas@tsc.upc.edu). diverse traffic granularity needs of the future Internet, the flexgrid elastic optical network (EON) architecture has been proposed $[2,3]$. By breaking the $50 \mathrm{GHz}$ rigid grid limit of conventional WDM networks, EONs increase the flexibility in connections provisioning. To do so, depending on the traffic volume, an appropriately sized optical spectrum portion is allocated to each connection. Therefore, incoming connection requests can be served in a spectrum-efficient manner.

Nevertheless, EON concept introduces additional challenges to the networking level, especially on the efficient connection establishment. Similar to WDM networks, an elastic optical connection must occupy the same spectrum portion between its end-nodes, that is, ensuring the so called spectrum continuity constraint. In addition, the entire bandwidth of the connections must be contiguously allocated, also referred as the spectrum contiguity constraint. The Routing and Spectrum Allocation (RSA) problem in flexgrid optical networks has been widely studied, with special emphasis in dynamic (in operation) network scenarios [3-6]. There, connection arrival and departure processes are random and the network has to accommodate incoming traffic in real time. In a dynamic scenario, the spectral resources allocated to connections are released upon tear down, becoming available for future requests. The randomness in the connections birth and death processes leads to fragmentation $[3,7]$ of the spectral resources in the network.

The spectrum fragmentation significantly decreases the probability of finding enough contiguous spectrum for establishing new incoming connections, especially those traversing multi-hop paths and/or requesting large amounts of bandwidth. In fact, new connection requests can be blocked in spite of having enough spectral resources if these are noncontiguous. Keeping spectrum fragmentation as low as possible is a major objective during flexgrid network operation. Nevertheless, fragmentation is intrinsic to the idea of dynamic flexgrid network, and different ways to reduce its effects have been extensively studied, mainly based on rearranging the fragmented spectrum [8-10]. The main idea behind defragmentation schemes consists in consolidating the available spectral fragments contiguously, so that they can be used for serving new incoming connection requests.

Spectral defragmentation reduces connections blocking 
probability, and then maximizes the service capacity of the network. In addition, it enables better network maintenance and more efficient network restoration procedures. While spectrum defragmentation improves the network spectrum utilization, connections reallocation increases the complexity and hence the cost of the network. Moreover, advanced optical devices such as wavelength converters and tunable optical switches are necessary for realizing such proposals, further increasing the complexity as well as the budget needed to deploy and operate the network. Finally, it is worth mentioning that a significant amount of control plane signaling messages are required to set up and release connections in the network, which burdens the control plane in the network with additional overhead. Minimizing connections disruption during defragmentation is a major challenge in flexgrid networks.

Most of the works about fragmentation have focused on reducing its impact once it is present. An alternative approach consists in trying to keep its value as low as possible during network operation. This work is focused on the optimization of link spectrum occupation during flexgrid network operation. If the fragmentation of the links spectrum is reduced, the amount of actions needed for rearranging it will be indeed less. To achieve this fragmentation reduction, a simple proposal consisting in limiting the elasticity of the offered connections is proposed, and its usefulness demonstrated analytically as well as by means of extensive simulations, both at the link and at the whole network level. Some previous works have dealt with the model of link spectrum use in flex-grid optical networks $[11,12]$. Both of them use Markov chains to derive analytical expressions that model the spectrum occupation in EONs. Nevertheless, these exact solutions require unaffordable computing resources in real scenarios (high capacity links and high traffic heterogeneity). Indeed, authors in [11] assess their model for a scenario with only two types of connections, and with a maximum capacity of 16 connections per link. On the other hand, authors in [12] derive an exact link model considering different EON characteristics (with or without spectrum contiguity constraint, random or first-fit assignment, with or without spectrum conversion), and provide approximations to the exact solution. They state that the approximation for the case without spectrum conversion, with contiguous spectrum assignment and first-fit assignment (WOSCWCSA-FF in [12]) only works for small capacity links. In this work we use their spectrum occupation Markov model but some differences are introduced:

- While in these previous works guard spectrum bands are considered part of the connections for the sake of simplicity, we put special emphasis on their impact on the network performance.

- The study is limited to a spectrum window with size close to the biggest allowed connection. The rationale behind this simplified model stems from the fact that this work focuses on the blocking causes, and once the path has been decided and the spectrum window assigned, the blocking is only possible when the window found is close to the connection size.

This work shows that, by limiting the allowed connections sizes to some selected values, some benefits are obtained. The rest of paper is organized as follows. Section II is devoted to the analysis of link spectrum occupation, and the main proposals about its optimization are introduced and discussed. Simulation results at the link level, assessing the previously introduced concepts, are presented in Section III. In Section IV simulations at the network level demonstrate that the concepts presented are useful when considering the whole network performance. Finally, Section V concludes the paper.

\section{LINK SPECTRUM OCCUPATION ANALYSIS}

To accommodate traffic in flexgrid networks, the total available spectrum is divided into constant spectrum units with a granularity finer than the typical $50-\mathrm{GHz}$ grid used in WDM systems. These spectrum units are referred as frequency slots (FS) and their typical width is $12.5 \mathrm{GHz}$, although other values such as $6.25 \mathrm{GHz}$ have also been proposed. If a 2 (or 4) $\mathrm{THz}$ spectrum is considered for a given link, the number of available FS is 160 (or 320). Each FS can carry some amount of traffic mainly depending on the modulation format used and the required reach [13]. For the sake of simplicity, this work just focus on the spectrum (number of FS) used, avoiding the spectral efficiency problem. Each connection is served by allocating a set of contiguous FS to it. In EONs, any number of FS (from 1 to $\mathrm{N}$ ) is allowed for connections. One FS is usually reserved as guard-band between adjacent connections. This implies that each connection, assuming that its required bandwidth is $\mathrm{BW}=\mathrm{n}$ FS, is actually occupying $\mathrm{n}+1$ FS. This simple assumption will be of great importance during the rest of this work.

It is easy to understand that the performance of the system clearly depends on the size (number of FS) of the connections. Let us see that by means of a simple example, illustrated in Figure 1, where it is also clear the simple and useful methodology introduced in this work that considers each connection as a set of n FS (colored blocks) plus a 1 FS (white blocks) for guard-band. Due to the randomness of connections arrivals and departures, some non-optimum spectrum usage situations can be reached. These states, shown in Figure 1, occur when there is a n+1 FS connection (including guard-band) allocated in an $n+2$ FS capable void, and are likely to occur when spectrum assignment strategy is First-Fit [14], which consists in placing the connection in the first capable (from left to right) spectrum void.

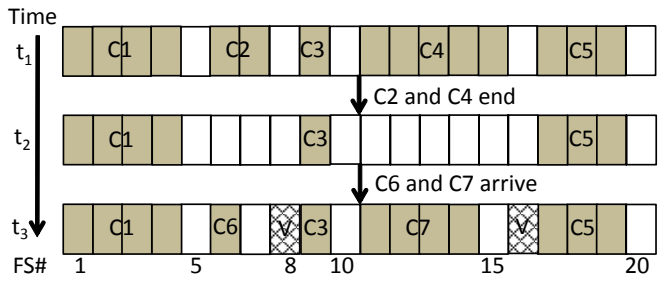

Fig. 1. Status of a $20 \mathrm{FS}$ spectrum window in an examplary link. At $\mathrm{t}=\mathrm{t}_{1}$, there are 5 live connections (C1 to C5). At $\mathrm{t}=\mathrm{t}_{2} \mathrm{C} 2$ and $\mathrm{C} 4$ have been torn down. At $\mathrm{t}=\mathrm{t}_{3} \mathrm{C} 6$ and $\mathrm{C} 7$ are set-up. FS 8 and FS 16 cannot be used while the current status is preserved.

In the example, the voids left by $\mathrm{C} 2$ (3 FS) and C4 (6 FS) are occupied, respectively, by C6 (2 FS) and C7 (5 FS). Once 
the connection is allocated, a $1 \mathrm{FS}$ void is created (V labelled cells in Figure 1), which is useless as the minimum bandwidth required to place a $1 \mathrm{FS}$ connection is $2 \mathrm{FS}$. It is intuitively deduced that this effect could be avoided by appropriately choosing the allowed sizes of the offered connections, and a key contribution of this work stems from this idea. An accurate study about this imperfection, as well as a simple strategy to overcome it, is offered in next paragraphs. The possible future events in an N FS spectrum window are analytically modeled as a time-homogeneous Markov chain with a finite states space [11, 12, 15], and its expected solutions are found. These states correspond, in our study, to the possible spectrum void sizes (from 1 to $\mathrm{N}$ FS, depending on the connections present), and transitions between them are triggered by connections arrival and departure.

Two different Markov chains are drawn in Figure 2, which helps to understand the problem modeling. In Figure 2.(a), a 5 FS spectrum window, where 1 to 4 FS connections can be allocated, is represented. This bandwidth window can be in 6 different states:

- F (Spectrum Full). Two combinations are possible: $14 \mathrm{FS}$ connection (see slots 1 to 5 in Fig. 1 at $\mathrm{t}=\mathrm{t}_{1}$ ), or $12 \mathrm{FS}+$ 1 FS connections (slots 6 to 10 in Fig. 1 at $t=t_{1}$ ). It is worth noting that the way how the different connections are ordered in the link spectrum has some impact on the system performance (a detailed study about that is given in [12]). Our model also takes into account the different permutations inside the spectrum window considered.

- $\mathrm{n}$ (with values $\mathrm{n}=1$ to 4 ). There is a spectrum void of $\mathrm{n}+1$ FS size, so a new connection (with $\mathrm{BW} \leq \mathrm{n}$ FS) could be allocated on it. If a connection death is the next event, the void size will increase (if $n=4$ the state won't change as this is the maximum size of interest).

- V (Void). An unusable void has been created. This situation is shown in FS 8 and FS 16 of Fig. 1 at $t=t_{3}(V$ labelled cells). In this case, it is not possible filling this void unless an adjacent connection is previously torn down. As has been remarked previously, a minimum of $2 \mathrm{FS}$ is needed to place any connection.

Figure 2.(b) represents a system where only odd values of FS per connection are allowed. In this case it is not possible that the system falls into the $\mathrm{V}$ state because new arriving connections always fit perfectly into the windows left (all the voids will have an even number of FS as required by the allowed connections).

For the sake of simplicity, draws in Figure 2 represent scenarios with a small number of connection sizes (4 in both cases). Nevertheless, the analytical solution to the problem has been found for an arbitrary window of N+1 FS. The rationale behind limiting the bandwidth window under study to the maximum size of the offered connections is obvious: when the First-Fit spectrum assignment strategy is applied, the portion of the spectrum involved in the problem, once the path has been decided, will always have this size at maximum. Therefore, for any given connection the assigned spectrum will be in this range. Conclusions about the behavior of a generic N+1 FS bandwidth window will give us insight into the performance of the link and therefore, about the whole flexgrid network behavior.

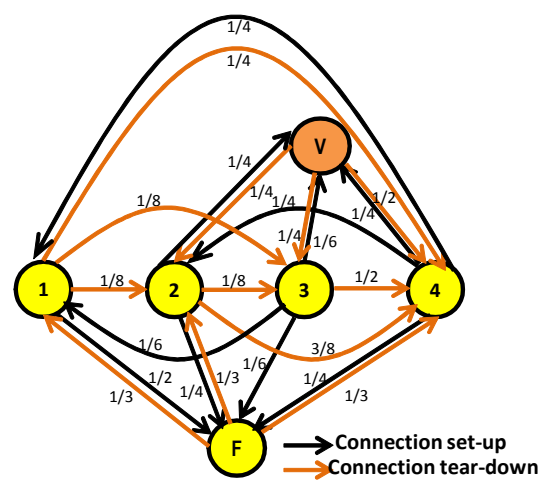

(a)

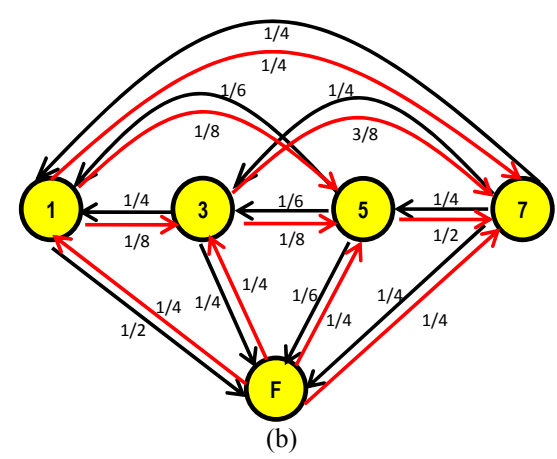

Fig. 2. Transition probabilities between different spectrum void sizes represented as a Markov chain. (a) 1, 2, 3 and 4 FS connections are allowed (the bandwidth window considered has 5 FS). (b) Only connections with odd $<8$ FS bandwidth values are generated (an 8 FS bandwidth window is considered in this case).

The Global Balance Equations (GBE) of the Markov chain are represented by the matrix in Figure 3, where the $\mathrm{N}+2$ possible states are indicated on top and on the left, and the matrix elements correspond to the probability values of changing from any state to any other.

\begin{tabular}{|c|c|c|c|c|c|c|c|c|c|}
\hline & F & V & 1 & 2 & $\ldots$ & $n$ & $\ldots$ & $N-1$ & $N$ \\
\hline$F$ & 0 & 0 & $\frac{F(N-2)}{F(N)}$ & $\frac{F(N-3)}{F(N)}$ & $\ldots$ & $\frac{F(N-1-n)}{F(N)}$ & $\ldots$ & 0 & $\frac{1}{F(N)}$ \\
\hline$V$ & 0 & 0 & 0 & $\frac{1}{N}$ & $\ldots$ & $\frac{1}{N}$ & $\ldots$ & $\frac{1}{N}$ & $\frac{2}{N}$ \\
\hline 1 & $\frac{1}{2}$ & 0 & 0 & $\frac{1}{2 N}$ & $\ldots$ & $\frac{1}{2 N}$ & $\cdots$ & $\frac{1}{2 N}$ & $\frac{3}{2 N}$ \\
\hline 2 & $\frac{1}{4}$ & $\frac{1}{4}$ & 0 & 0 & $\ldots$ & $\frac{1}{2 N}$ & $\ldots$ & $\frac{1}{2 N}$ & $\frac{4}{2 N}$ \\
\hline$\ldots$ & $\ldots$ & $\ldots$ & $\ldots$ & $\ldots$ & 0 & $\ldots$ & $\ldots$ & $\ldots$ & $\ldots$ \\
\hline$n$ & $\frac{1}{2 n}$ & $\frac{1}{2 n}$ & $\frac{1}{2 n}$ & $\frac{1}{2 n}$ & $\ldots$ & 0 & $\ldots$ & $\frac{1}{2 N}$ & $\frac{n+2}{2 N}$ \\
\hline$\ldots$ & $\ldots$ & $\ldots$ & $\ldots$ & $\ldots$ & $\ldots$ & $\ldots$ & 0 & $\ldots$ & $\ldots$ \\
\hline$N-1$ & $\frac{1}{2(N-1)}$ & $\frac{1}{2(N-1)}$ & $\frac{1}{2(N-1)}$ & $\frac{1}{2(N-1)}$ & $\ldots$ & $\frac{1}{2(N-1)}$ & $\ldots$ & 0 & $\frac{N}{2 N}$ \\
\hline$N$ & $\frac{1}{N}$ & $\frac{1}{N}$ & $\frac{1}{N}$ & $\frac{1}{N}$ & $\cdots$ & $\frac{1}{N}$ & $\ldots$ & 0 & 0 \\
\hline
\end{tabular}

Fig. 3. Markov GBE matrix representation for an N+1 FS bandwidth window. $F(i)$ terms in the matrix elements correspond to the $\mathrm{i}^{\text {th }}$ term of de Fibonacci succession

By solving the GBE, with the additional equation $\mathrm{F}+\mathrm{V}+1+2+\ldots+\mathrm{n}+\ldots+(\mathrm{N}-1)+\mathrm{N}=1$, which implies that the 
system has to be necessarily in one of these states, the expected performance of the system in the steady state is found. In the example shown in Figure 2.(a), these N+2 states correspond to the voids capable of $1 \mathrm{FS}$ to $4 \mathrm{FS}$ connections, the $\mathrm{F}$ state and the $\mathrm{V}$ state.

To determine the matrix elements it has been considered that at any moment a connection can be either generated or torn-down with probability $1 / 2$. Of course, bandwidth of new connections is limited to values smaller or equal than the void size. On the other hand, the birth and death events assume uniform probability distribution in the sizes of allowed connections. Obviously, only existing connections can be torn down at any given instant. Following this rationale it has been found that the set of connections permutations that can be allocated in an N FS window are determined by the Fibonacci succession. Then, the $F(i)$ terms in the matrix correspond to the $i^{\text {th }}$ terms of de Fibonacci succession. The reader is referred to [16] for a detailed study on this result. This interesting outcome opens the door to new ideas on suitable EON traffic profiles, taking into account the special properties of the $\mathrm{F}(\mathrm{i})$ numbers, that are left for future study.

Let us go back to Figure 2.(a) to better understand the principles applied in the derivation of the GBE (whose values are given in left matrix of Figure 4):

- System is in the F state. Only connection death is possible. This is a special state because only a limited set of connections combinations lead to it. Concretely, one $4 \mathrm{FS}$, or one $1 \mathrm{FS}$ plus one $2 \mathrm{FS}$ connections are the only possibilities if $\mathrm{N}=4$, as shown in FS 1 to 5 and 6 to 10 of Fig. 1 at $t=t_{1}$. There are then three different combinations leading to $\mathrm{F}$ for $\mathrm{N}=4: 4 \mathrm{FS}, 2 \mathrm{FS}+1 \mathrm{FS}$, and $1 \mathrm{FS}+2 \mathrm{FS}$. The fourth term of Fibonacci succession is just 3. Therefore, the arrows departing from $\mathrm{F}$ go to states 1,2 and 4 with probability $1 / 3$. If, for example, $\mathrm{N}=8$ is examined, it is found that 13 different combinations lead to $\mathrm{F}(7,5+1,1+5,2+4,4+2,3+3$, $3+1+1,1+3+1,1+1+3,2+2+1,2+1+2,1+2+2,1+1+1+1)$. Indeed, 13 is the $8^{\text {th }}$ term of the Fibonacci succession. Assuming that the system is in one of these states, the probability of death of each one of the bandwidth values (n from 1 to 7 ) is $F(8-n)$, as represented in Figure 3 matrix.

- System is in states 1 to 4 . Taking into account again the extra FS used for guard-band, these states correspond to spectrum windows with size 2, 3, 4 or 5 FS. Each state has its particular states transitions. Let us examine state 3 as an example. First of all, next event being a birth or a death is considered equally probable. If it is a death the system will go necessarily to state 4 (its size was $3 \mathrm{FS}$ before this death), so the probability of going to 4 is $1 / 2$. If it is a birth, it is equally probable that the new connection is 1 FS (next state would be 1), 2 FS (next state would be V) or 3 FS (next state would be F). Therefore, the probability associated to each one of these exiting arrows (black arrows in the Figure) is $1 / 6$. State 4 is special as it has been considered that only connection birth is possible from this state (it is symmetric with respect to state F).

- System is in state V. Only death is possible from this state (as has been explained previously, no connection can be allocated when the system is in the V state). In this case, if the new connection needs 1FS (this event has probability $1 / 4$ ), the system goes to state 2 . If it is 2 FS (also with probability $1 / 4$ ) the system moves to state 3 . Finally, if the new connection size is 3 or $4 \mathrm{FS}$, the system goes to state 4 (probability of this event is $1 / 2$ ).

After solving the GBE for a generic N FS capable window, it has been found that the probability of the system falling into the $\mathrm{V}$ state, which can cause wasting spectrum, is $8.9 \%$ when $\mathrm{N}=8$ ( 8 will be the maximum number of FS per connection used during simulations in the fully elastic case). These equations have also been solved for higher spectrum window values and the final conclusion is that $\mathrm{V}$ state is really likely to appear. Likelihood results for any $\mathrm{N}$ value are ordered from higher to lower values as void sizes range from 1 FS (V state) to (N-1) FS. Expected probabilities of falling into the $\mathrm{V}$ state for different $\mathrm{N}$ values, found by solving the GBE, are given in next Table.

TABLE I

\begin{tabular}{|c|c|c|c|c|c|c|}
\multicolumn{7}{c}{ PROBABLILITY OF V STATE } \\
\hline N & 8 & 9 & 10 & 12 & 14 & 16 \\
\hline Prob(V) & 0.089 & 0.085 & 0.081 & 0.075 & 0.071 & 0.067 \\
\hline
\end{tabular}

Probability of falling into the $\mathrm{V}$ state for spectrum windows of $\mathrm{N}+1$ FS and assuming that any connection from $1 \mathrm{FS}$ to N FS are equally probable.

It has to be highlighted nonetheless that being in the $\mathrm{V}$ state does not imply directly wasting spectrum, as transition to other states will occur sooner or later. Nevertheless, it produces at least some harmful instantaneous effect.

The model shown in Figure 2.(b), represents a system where only connections with odd number of FSs are allowed. In this case, there is no probability that the system falls into the $\mathrm{V}$ state, as all the allowed connections (with their corresponding guard-band FS) occupy an even number of FS. Therefore, from the spectrum efficient use point of view, limiting the connections sizes to odd FS values (referred as Odd scheme in next Sections) seems to be a good idea. When compared to elastic connections bandwidth values, ranging from 1 to N FS, other combinations are also advantageous. It has been intuitively deduced that, if the allowed number of FS per connection is limited to 2, 5 and 8 (named 2-5-8 scheme in next Sections), the chances of falling into the $\mathrm{V}$ state are also eliminated, as, taking into account guardband slots, all the voids created will be multiple of 3 . These pseudo-elastic models where only odd (from 1 to 9FS), or 2, 5 and 8 FS per connection are allowed, (so they should be better classified as multi-rate instead of elastic) will be evaluated by simulation in Section III, and their efficiency concerning link spectrum occupation will be assessed.

As a final contribution on this point, particularizations of the Figure 3 GBE matrix for the examples in Figure $2(\mathrm{~N}=4$ and $O d d$ ), as well as for the 2-5-8 case, are given in Figure 4. It can be seen in Figure 2 (left matrix) that falling into the $\mathrm{V}$ state when the void is equal or larger than $2 \mathrm{FS}$ is really likely (probability ranges from $1 / 6$ to $1 / 4$ ). 


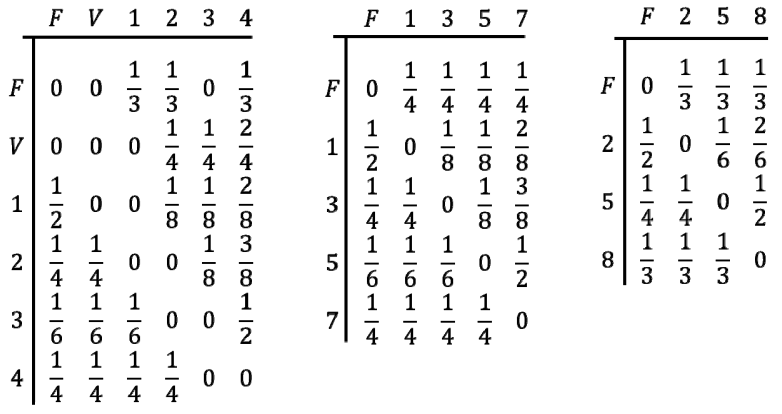

Fig. 4. Matrices representing the Markov GBE for the examples shown in Figure 2, as well as for the multi-rate 2-5-8 proposal.

Looking at the spectrum usage problem from another point of view, it is worth noting that the effective capacity of connections (EC) also depends on their bandwidth (BW) as a connection with $\mathrm{BW}=\mathrm{n}$ FS is actually occupying $\mathrm{n}+1$ FS. Therefore, EC for a given n FS connection is defined as,

$$
E C=\frac{n}{n+1}
$$

The average EC can then be found as the product of the EC and the probability of appearance of every type of connections. EC values have been computed for all the different traffic profiles studied in this work and are given in Table II of next Section. It is concluded from that Table that EC values clearly depend on the profile of the offered traffic. As an example, an additional gain of $6.6 \%$ (0.7889 vs. 0.74 ) is obtained when using the $2-5-8$ scheme with respect to the Elastic case for TP-3. This further enhances the advantage obtained by limiting the connections BW to these selected values, and helps in compensating for the flexibility lost when excluding some BW values. With the aim of carrying out a fair comparison, this effect will be removed during simulations by properly defining the maximum link load. It is shown in [17] that the lack of flexibility, which is the main weakness of the $2-5-8$ scheme, can be overcome by appropriately mapping the connections. As an example, allocating two 2 FS connections when one 4 FS connection is required would be the solution, but using this strategy would degrade the EC value, as 6 FS would be necessary in the $2-5-8$ case, while only 5 FSs are used in the Elastic one. A ceiling case with overprovisioning of FS is also considered during next Section simulations with the purpose of a worst-case comparison.

\section{Simulation RESUlTS}

As has been stated in previous Section, randomness in EON connections birth and death can bring the spectrum to inefficient states, where useless voids are created (V state). It has been shown that creating these $\mathrm{V}$ states is likely to happen and that they can be avoided by appropriately limiting the sizes of the connections. With the purpose of quantifying the benefits obtained when avoiding the $\mathrm{V}$ state, an ad-hoc Matlab® simulator has been developed. Its main characteristics are:

- Maximum number of FS per link equal to 160. This is a typical value used in previous works, and it has no special relevance on the results obtained.

- Number of FS per connection (BW) ranging from 1 to 8 is considered as the Elastic case. It is compared with Odd case (only odd BW values from 1 to 9 FS are allowed), and the 2-5-8 case (connections sizes are limited to 2, 5 and $8 \mathrm{FS}$ ). It has to be highlighted that, due to network fragmentation, larger connections experience always higher blocking, as finding enough contiguous FS becomes harder as connections size increases [18]. Therefore, the Odd case has a weakness from this point of view (connections with BW=9 FS are only present in this case). On the other hand, the average connections size is 5 FS for Odd and 2-5-8 cases, while it is 4.5 FS for the Elastic case. The Elastic case has some advantage here as smaller connections will be generated in average. On the other hand, a lower average bandwidth implies a lower EC value (more FS are wasted for guard-band), but this effect is compensated by appropriately defining (see eq. (3)) the maximum link load.

- Connections inter-arrival time (IAT) follows a Poisson process with average value $\overline{I A T}=1$. Connections holding time (HT) follows a negative exponential distribution. The average value $(\overline{H T})$ is adjusted to obtain the appropriate Offered Load values (those that led the bandwidth Blocking Probability to the desired values).

The load offered to the link is defined as,

OfferedLoad $=\frac{\overline{H T} * \overline{B W}}{\overline{I A T} * \text { MaxLoad }}$

where the maximum link load (MaxLoad) is defined as,

$$
\text { MaxLoad }=160 * \frac{\overline{B W}}{\overline{B W}+1}
$$

The use of one FS as a guard-band associated to each one of the connections is considered here again. It has to be noted that in a perfectly full spectrum (with size $160 \mathrm{FS}$ ), the number of allocated connections could range from 80 (all of them having BW=1 FS, so the effective load would be 80 FS) to 16 (if they are assumed with BW=9 FS, effective load in this case would be $144 \mathrm{FS})$. By defining the offered load using eqs. (2) and (3), the differences in EC (introduced in Section II, and whose numerical values are given in Table II) are compensated, and the effects observed during simulations are just due to the different spectrum usage strategies.

To assess the validity of the results obtained during simulations, different traffic profiles have been considered, (they are summarized in Table II):

- TP1. Corresponds to the uniform distribution. At the generation stage, each connection can take any of the allowed BW values with uniformly distributed probability. As a ceiling case used for benchmarking, a special mapping of Elastic to Multi-rate connections with spectrum overprovisioning is also considered (see 2-5-8 Ovp and Odd Ovp in Table II). In this case, the required bandwidth is always rounded up to the next allowed size (e.g. a 5 FS connection is set-up when the incoming demand needs 3 FS in the 2-5-8 Ovp model). 
- TP2. Gaussian distribution where the central values (BW near 5 FS) are more likely to appear. The generation of the $\mathrm{BW}$ values follows a normal distribution with mean 5 and standard deviation 2.5. Indeed (and this also applies to TP3), once each random value has been generated, the closest allowed BW value is selected.

- TP3. Also normal distribution but, in this case, the mean value is 5 and the standard deviation is set to 6.5 , making the extreme values more likely to appear than those near the average, because values below 1 result in a 1 FS connection, and a 8 FS connection is generated when 8 is surpassed.

\section{TABLE II}

OfFEREd TRAFFic Profiles

\begin{tabular}{|c|c|c|c|c|c|c|c|c|c|c|c|}
\hline & & \multicolumn{9}{|c|}{$\%( \pm 1)$ of Connections with \# FS } & \multirow[t]{2}{*}{ EC } \\
\hline & \# & 1 & 2 & 3 & 4 & 5 & 6 & 7 & 8 & 9 & \\
\hline \multirow{5}{*}{ TP1 } & Elastic & 12.5 & 12.5 & 12.5 & 12.5 & 12.5 & 12.5 & 12.5 & 12.5 & - & 0.7714 \\
\hline & Odd & 20 & - & 20 & - & 20 & - & 20 & - & 20 & 0.7717 \\
\hline & $2-5-8$ & - & 33 & - & - & 33 & - & - & 33 & - & 0.7963 \\
\hline & $\begin{array}{l}\text { Odd } \\
\text { Ovp }\end{array}$ & 12.5 & - & 25 & - & 25 & - & 25 & - & 12.5 & 0.7094 \\
\hline & \begin{tabular}{|c|c|}
$2-5-8$ \\
Ovp \\
\end{tabular} & - & 25 & - & - & 37.5 & - & - & 37.5 & - & 0.6667 \\
\hline \multirow{3}{*}{ TP2 } & Elastic & 7 & 9 & 15 & 19 & 19 & 15 & 9 & 7 & - & 0.7874 \\
\hline & Odd & 11 & - & 23 & - & 32 & - & 23 & - & 11 & 0.7944 \\
\hline & $2-5-8$ & - & 25 & - & - & 50 & - & - & 25 & - & 0.8056 \\
\hline \multirow{3}{*}{ TP3 } & Elastic & 28 & 7 & 7 & 8 & 8 & 7 & 7 & 28 & - & 0.7400 \\
\hline & Odd & 32 & - & 12 & - & 12 & - & 12 & - & 32 & 0.7430 \\
\hline & $2-5-8$ & - & 40 & - & - & 20 & - & - & 40 & - & 0.7889 \\
\hline
\end{tabular}

TP1 corresponds to Uniform distribution; TP2 and TP3 are Gaussian distributions. Values in white cells are percentages of appearance of every size (column values represent 1 to 9 FS connection bandwidth size). Last column values correspond to average effective capacity (EC) for the different cases

As was stated in Section II, this work focuses on improving link spectrum occupation by using special characteristics of the flexgrid connection requests. The link spectrum assignment (SA) strategy used is First-Fit, (connections are allocated as on the left of the spectrum as possible), which has been demonstrated as a good strategy to keep fragmentation at reasonable values [14]. The main idea behind First-Fit is keeping the spectrum as compact as possible, so more unused FS will be available for future connection requests. On the one hand, by using this strategy we are promoting maximum re-use of the spectrum in the network, which is a key objective to improve EON performance. On the other hand, spectrum fragmentation is somehow minimized as existing voids will be occupied as soon as possible. As discussed in Section II, First-Fit can nevertheless lead the spectrum window to the $\mathrm{V}$ state, which can be harmful for the link and whole network performance. In summary, and taking indeed into account that First Fit can undergo some drawback with time varying connections [7], it is usually assumed as an optimum SA policy in EONs.

Once all the parameters in the simulation framework are fixed, Figure 5 summarizes the results obtained in terms of bandwidth blocking probability (BP) versus load offered to the link. The different traffic profiles as well as the different spectrum occupation strategies have been simulated. Some clear conclusions can be extracted from these results. The most important one is that, as expected after the theoretical analysis, the 2-5-8 model clearly overcomes the rest in terms of BP. This is not surprising as it assumes a multi-rate (not completely elastic) optical network. As was stated in Section II, limiting the possible connections sizes makes the network more robust against fragmentation, especially when the allowed sizes satisfy some requirements (the bandwidth sizes used in this case avoid falling in the $\mathrm{V}$ state). It can be concluded that the gain obtained, in terms of $\mathrm{BP}$, is considerable. If we look at $\mathrm{BP}=10^{-2}$ and consider the Offered Load values leading to this $\mathrm{BP}$ in the different cases, we see that the improvement in terms of supported link load with respect to the Elastic case goes from a very small gain when Odd and TP-1 are used (both graphs actually cross at this point and Elastic overcomes Odd), to a maximum gain of $5.5 \%$ which is obtained when the $2-5-8$ case and TP3 are considered.

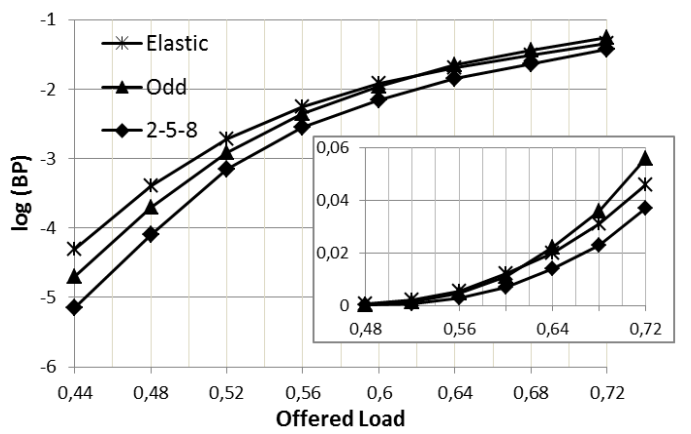

(a)

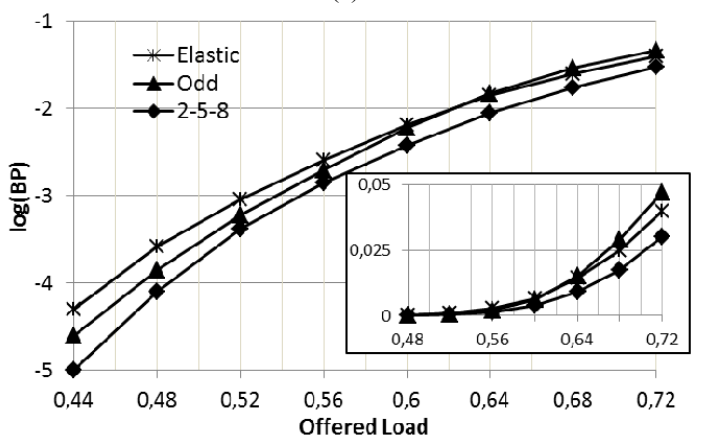

(b)

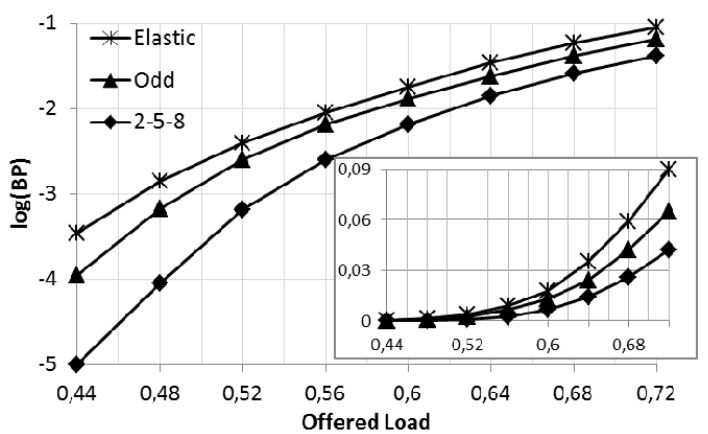

(c)

Fig. 5. Bandwidth blocking probability (BP, represented in logarithmic scale and linear scale in the insets) vs. Offered Load for the three spectrum occupation schemes considered, and the different traffic profiles: (a) TP1, (b) TP2 and (c) TP3.

Although these gains could appear to be minor, 
considering that the offered load has been normalized to its maximum as expressed in Eqs. (2) and (3), a gain of 5.5\% actually supposes an average additional effective load of 7.33 FS (more than $90 \mathrm{GHz}$ ) used in the link at the same bandwidth BP.

After analyzing these results, it could be argued that they are quite obvious because using only three (2-5-8 case) or five (Odd case) bandwidth values is clearly advantageous in front of the Elastic case where all the sizes are offered. Figure 6 shows de results obtained when Elastic is compared to 2-5-8 Ovp and Odd Ovp.

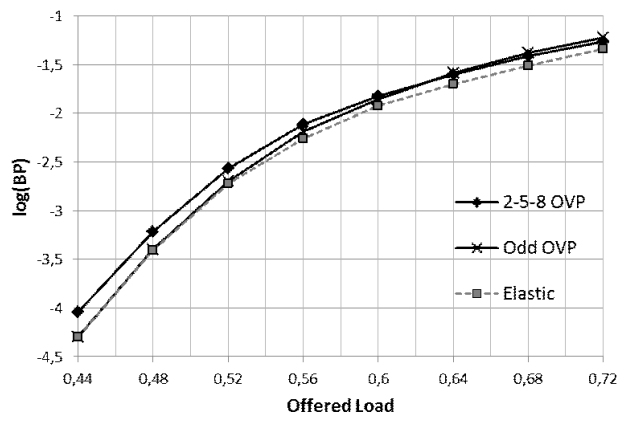

Fig. 6. Blocking probability (BP) versus Offered Load. Traffic profile assumed is TP- 1 (rows 1,4 and 5 of Table II). Actual supported load in 2-5-8 and Odd cases is higher due to overprovisioning.

It can be seen how in this case the BP is higher for the multi-rate schemes. Nevertheless, it has to be taken into account that many additional FS are allocated in these cases. The actual load supported by the 2-5-8 Ovp case is at least a $15 \%$ higher than that of the Elastic case. This means that there are plenty of allocated and unused FS, which could be utilized in case of time varying connections (the connection required BW is increased during its HT) or even if new small connections arrive.

As a final step to demonstrate that the avoidance of the $\mathrm{V}$ state is beneficial, simulations comparing the performance of the 2-5-8 case with the 2-5-7 one (connections sizes limited to 2, 5 or 7 FS) were also carried out. Figure 7 shows the obtained results.

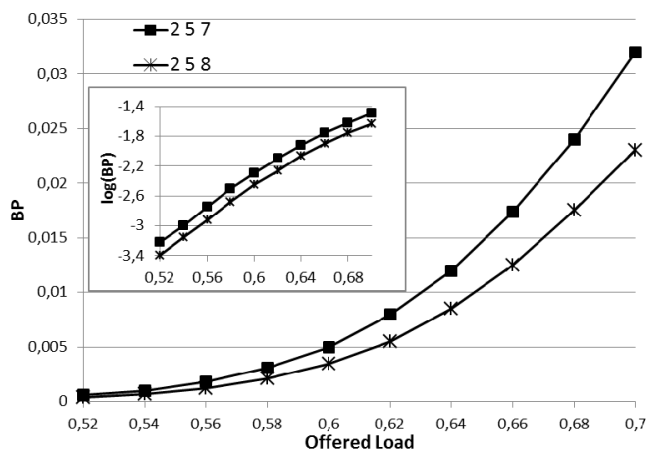

Fig. 7. Blocking probability (BP) versus Offered Load (using TP2 ), for two different multi-rate scenarios. The $2-5-8$ case outperforms the 2-5-7 one, as there is no possibility of having 2 FS voids. The inset shows the logarithmic representation of the results obtained.

It has to be taken into account that the 2-5-7 distribution should experience lower BP as larger bandwidth connections always suffer higher BP in flexgrid networks. The results obtained confirm that the strategy proposed (avoiding the $\mathrm{V}$ state to reduce the $\mathrm{BP}$ ) works, as the performance of the 2-5-8 system is superior even considering its drawback using larger connection BW values. Considering TP2 (normal distribution on connections bandwidth with average 5 and standard deviation 2.5), the average size value for the 2-5-7 case is 4.67 (in front of 5 for the 2-5-8 case). To compensate this difference, the HT/IAT value has been appropriately adjusted, ensuring that the offered load (applying again eq. (3) definition) is identical in both cases. It is concluded from Figure 7 that the gain obtained holds even when the 2-5-8 case is compared with this also limited set of eligible bandwidth values (as in the 2-5-8 case, only three different bandwidth values are allowed). Looking again at $\mathrm{BP}=10^{-2}$, it is shown that the load supported in the $2-5-8$ case is $2 \%$ higher, so in average, 2.7 additional FS would be allocated in the link at this BP value when using the 2-5-8 combination. The advantage of avoiding the $\mathrm{V}$ state is therefore undoubtedly demonstrated.

In order to better quantify the effect of avoiding the $\mathrm{V}$ state in the link spectrum, the number of spectrum voids in the link has been monitored during simulations. Graphs in Figure 8 are exemplary of the results obtained. While in the Elastic case the average number of voids during simulations is around 7, this number is reduced to average values below 5.5 and 4.5 when considering, respectively, the $O d d$ and 2-58 cases. Obviously, these values experienced some fluctuations when changing the offered load and traffic profile, but those indicated above are certainly representative.

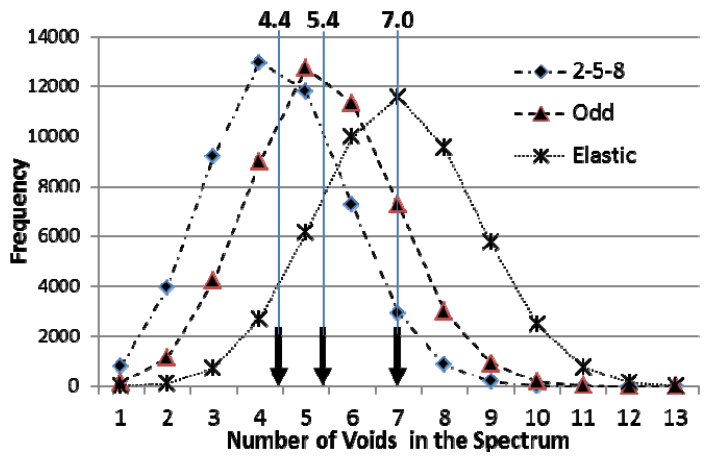

Fig. 8. Frequencies obtained for the different number of voids in the link during a given $10^{5}$ connections simulation. The traffic profile is TP-1, and the offered load is 0.6 (Bandwidth blocking probability values are around $1 \%$ ). Values over the arrows are the average number of voids in the link during the whole simulation (2-5-8, Odd and Elastic cases from left to right).

Let us consider an existing spectrum void $\mathrm{A}$ at a given instant, with size N+1 FS (so it is able to allocate an N FS connection). It has been created when one of the previously existing connections has terminated. If a new connection request arrives, it can only be allocated in $\mathrm{A}$ if its $\mathrm{BW} \leq \mathrm{N}$. Considering our spectrum allocation policies, in the Elastic case they are 8 different connection sizes (from 1 to $8 \mathrm{FS}$ ), while there are 5 options for Odd case, and only 3 different sizes for the $2-5-8$ case. It is easy to see that the probability of using $\mathrm{A}$ in a generic case when the number of possible 
connection sizes is $\mathrm{K}$ will be given by,

$$
P(\text { use } A)=\frac{1}{K} \sum_{n=1}^{K} \frac{n}{K}=\frac{K+1}{2 K}
$$

Therefore, if we use $\mathrm{K}=3,5$ and 8 , (which are the number of sizes allowed, respectively, in the 2-5-8, Odd and Elastic cases), we conclude that at any given instant the probability of using an existing void is $33 \%$ higher in the best case (2-58) compared to the worst one (Elastic). This effect is indeed magnified if we take into account that Elastic case has some probabilities of going to the useless $\mathrm{V}$ state (as explained in Section II), while this is avoided in the Odd or the 2-5-8 cases. Therefore, the average number of spectrum voids in the Elastic case is $59 \%$ higher than that in the 2-5-8 (7 vs. 4.4 in Figure 7), having a clearly more fragmented spectrum in the former case.

With the purpose of proving the likeliness of the appearance of the $\mathrm{V}$ state shown in Table I as the result of the analytical study, the number of voids with size equal to 2 FS (corresponding to the $\mathrm{V}$ state) has also been monitored during simulations. It has been found that around $11 \%$ of the spectrum voids have this size. The expected theoretical value given in Section II when $\mathrm{N}=8$ (see Table I) is $8.9 \%$. It can be concluded that the simulation results assess the validity of the theoretical model. The slight increase obtained can be due to the effect of First-Fit assignment strategy which is avoided in the model.

All in all, it has been extensively proven that, by sacrificing some flexibility (avoiding some connection BW values in the flex-grid network), a substantial benefit in terms of carried traffic for a given bandwidth BP is obtained. Furthermore, different modulation formats lately proposed give an additional degree of freedom to accommodate different bit rates on a given bandwidth. For example, (see [3, 13] for detailed analysis) a 2 FS connection can convey capacities from $<40 \mathrm{Gbit} / \mathrm{s}$ (when binary modulation formats are used) to $>100 \mathrm{Gbit} / \mathrm{s}$ (if complex multi-level modulation formats and coherent detection are present). On the other hand, if the Odd case is considered, elastic to multi-rate connections mapping schemes have been proposed [17], which allow combining different connections to attain even FS values. The proposal has obviously a clear weakness in the flexibility lost (connection sizes are limited). On the other hand, allocating for example 5 FS when only 3 FS are requested (as has been assumed in the cases with overprovisioning) can be beneficial in the long term if new connections have the same source and destination, or simply the BW requested by the connection increases during its lifetime. However, these effects are left for future study as the purpose of this work is to formulate an efficient way of distributing connections in a flexgrid network.

\section{LINK AND NETWORK PERFORMANCE CORRELATION}

When reviewing previous contributions on spectrum utilization in flexgrid optical networks [12, 19-21], the focus is usually on the whole network performance. For the sake of simplicity and clarity, this work has analyzed the problem on a single link, so the spectrum contiguity constraint is avoided. Nonetheless, in this section the simulations have been extended to the whole network, in order to assess that the benefits obtained at the link level hold.

Considering the NSFnet (14-Nodes 21-Links) and the European Optical Network (EONet, 19-Nodes 37-Links) topologies (both shown in Figure 9), the correlation between link blocking probability and network blocking probability has been analyzed, assuming the same traffic parameters given in previous Section.

As a first step, the frequency use of each of the links has been measured. It has been found that, using a simple Shortest Path routing algorithm, the NSFnet IL-PA link is used by roughly $8 \%$ of the connections. When analyzing the EONet, again some selected links carry a considerable amount of traffic. For example, link 5-9 (Par-Mil) is utilized by more than $8 \%$ of the connections during simulations.

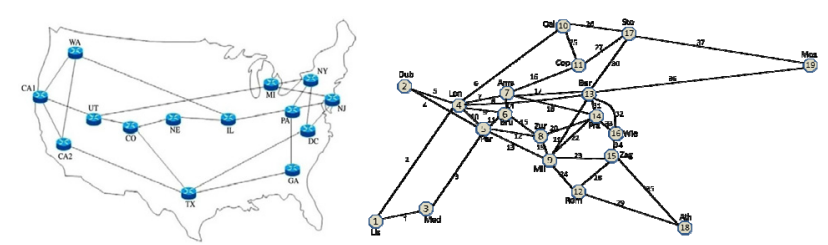

Fig. 9. Topologies of the NSFnet and EONet networks, used during simulations.

The load offered to the links has been normalized taking into account the average number of hops (NHops) of established connections, so equation (2) now becomes,

$$
\text { MaxLoad }=\frac{160}{\text { NHops }} * \frac{\overline{B W}}{\overline{B W}+1}
$$

The fact that each connection spreads over NHops links is therefore taken into account. This fact actually explains why Offered Load per link necessary to reach a certain BP value is lower when the EONet is considered. Other parameters used during simulations are:

- Traffic profiles are those shown in Table I.

- Source-Destination pairs are uniformly distributed among all the networks' nodes.

- Regarding the routing and spectrum allocation algorithm for allocating new connections, simulations run a typical $\mathrm{k}$-Shortest Path (with $\mathrm{k}=3$ ) routing and First-Fit SA [20].

- The connections bandwidth generation policies follow the schemes previously introduced (Elastic, Odd and $2-5-8$ ) as the main motivation of this work is just on this point.

Results obtained during simulations assess the validity of Section II discussions. It is again demonstrated, now at the network level, that the strategy of sacrificing some flexibility provides additional advantage in terms of supported network load. The obtained results are summarized in Figure 10, where it can be observed how performance of the 2-5-8 scheme is clearly superior to the alternative connections generation schemes. Looking again at the $\mathrm{BP}=10^{-2}$ point, load supported by the $2-5-8$ scheme is around $4 \%$ higher than that supported by the other schemes. 


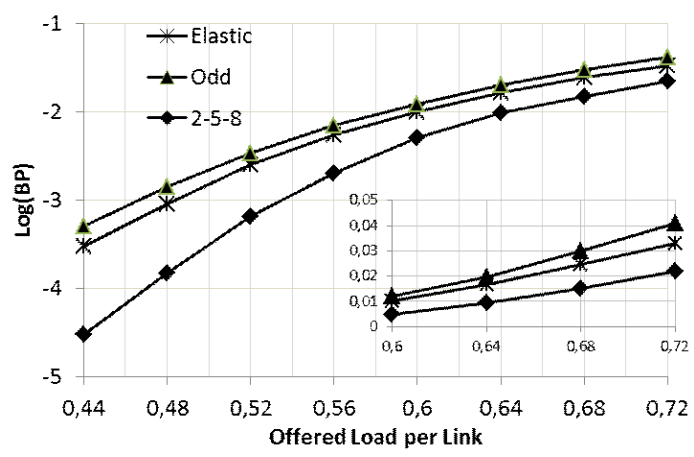

(a)

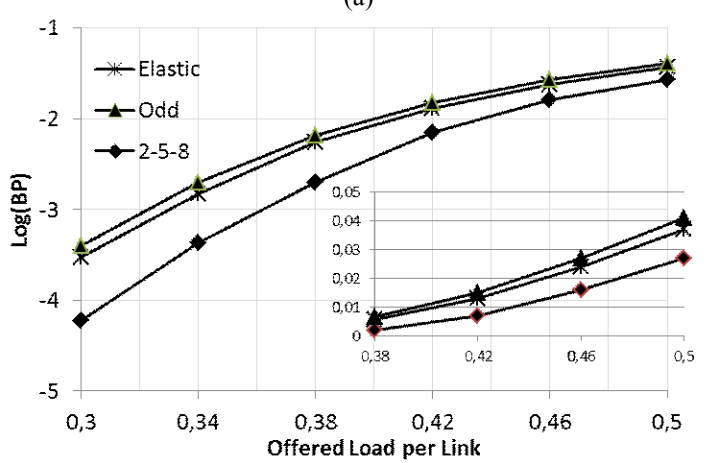

(b)

Fig. 10. Bandwidth blocking probability at the network level vs. Average Offered Load per link. (a) NSFnet, (b) EONet. Uniformly distributed connections bandwidth (TP-1) has been used in the case shown. Similar performance has been observed for TP-2 and

TP-3. Graphs in the insets show linear BP values.

When comparing the Elastic and Odd cases at the network level, their performance is almost equal (Elastic is slightly better). At the link level Odd outperformed Elastic only for very low load values. As has been already commented, using 9 FS connections implies that finding enough free voids becomes more difficult as load increases.

It can be therefore concluded that the results obtained in this work at the link level can be extrapolated to the flexgrid whole network performance. In summary, the thorough study of the link performance has some intrinsic value and gives insight into spectrum use strategies to be followed to optimize flexgrid networks performance.

\section{Conclusion}

Flexgrid optical network arises as a promising solution for future high-speed optical transmission, since it can provide superior flexibility and scalability in spectrum allocation, supporting the diversity of services along with the rapid growth of Internet traffic. This paper introduces some strategies to improve spectrum usage in flexgrid networks, which reduce the link fragmentation. The effects of fragmentation in the effective spectrum utilization have been analytically modeled, and it has been proven that these effects can be decreased by limiting the allowed connections bandwidth sizes. By means of simulation, it has been demonstrated that such a proposal reduces blocking in the network, as link fragmentation is considerably reduced. Gains up to $5 \%$ in effective link capacity have been obtained by applying the strategies proposed. When considering the whole network performance, gains around $4 \%$ in supported load for a $\mathrm{BP}=10^{-2}$ have been obtained. These gains are even enhanced because the spectrum utilization of the new proposed schemes, measured as the number of FS carrying traffic, is actually higher.

\section{ACKNOWLEDGMENT}

This work has been supported by the Government of Catalonia (SGR2014-1254) and the Spanish MICINN (SINERGY project, TEC2014-59995-R).

\section{REFERENCES}

[1] Cisco White Paper, "Cisco Visual Networking Index: Forecast and Methodology, 2013-2018", 2014.

[2] M. Jinno, H. Takara, B. Kozicki, Y. Tsukishima, Y. Sone, and S. Matsuoka, "Spectrum-Efficient and Scalable Elastic Optical Path Network: Architecture, Benefits, and Enabling Technologies," IEEE Commun. Mag., vol. 47, no. 11, pp. 66-73, 2009.

[3] O. Gerstel, M. Jinno, A. Lord, B. Yoo, "Elastic Optical Networking: A New Dawn for the Optical Layer," IEEE Commun. Mag., vol. 50, no. 2, pp. s12-s20, 2012.

[4] K. Christodoulopoulos, I. Tomkos, E. Varvarigos, "Dynamic Bandwidth Allocation in Flexible OFDM-based Optical Networks," in Proc. OFC, 2011, OTuI5.

[5] T. Takagi, H. Hasegawa, K. Sato, Y. Sone, B. Kozicki, A. Hirano, M. Jinno, "Dynamic Routing and Frequency Slot Assignment for Elastic Path Networks that Adopt Distance Adaptive Modulation," in Proc. OFC, 2011, OTuI7.

[6] A. Castro, L. Velasco, M. Ruiz, M. Klinkowski, J. FernándezPalacios, D. Careglio, "Dynamic Routing and Spectrum (Re) Allocation in Future Flexgrid Optical Networks," Computer Networks, vol. 56, no. 12, pp. 2869-2883, 2012.

[7] P. S. Khodashenas, J. Comellas, S. Spadaro, J. Perelló, G. Junyent, "Using Spectrum Fragmentation to Better Allocate Time-Varying Connections in Elastic Optical Networks", J. Opt. Commun. Netw., vol. 6, no. 5, pp. 433-440, 2014.

[8] T. Takagi, H. Hasegawa, K. I. Sato, Y. Sone, A. Hirano, M. Jinno, "Disruption Minimized Spectrum Defragmentation in Elastic Optical Path Networks that Adopt Distance Adaptive Modulation," in Proc. ECOC 2011, Mo.2.K.3.

[9] F. Cugini, F. Paolucci, G. Meloni, G. Berrettini, M. Secondini, F. Fresi, N. Sambo, L. Poti, P. Castoldi, "Push-Pull Defragmentation Without Traffic Disruption in Flexible Grid Optical Networks", J. Lightw. Technol., vol. 31, no. 1, pp.125133, 2013.

[10] X. Yu, J. Zhang, Y. Zhao, T. Peng, Y. Bai, D. Wang, X. Lin, "Spectrum Compactness based Defragmentation in Flexible Bandwidth Optical Networks", in Proc. OFC/NFOEC 2012, JTh2A.35.

[11] Y. Yu, J. Zhang, Y. Zhao, X. Cao, X. Lin, W. Gu, "The First Single-Link Exact Model for Performance Analysis of Flexible Grid WDM Networks", in Proc. OFC/NFOEC 2013, JW2A.68.

[12] H. Beyranvand, M. Maier, J. A. Salehi, "An Analytical Framework for the Performance Evaluation of Node- and Network-Wise Operation Scenarios in Elastic Optical Networks", IEEE Transac. on Comms, vol. 62, no. 5, pp. 1621$1633,2014$.

[13] I. Popescu, I. Cerutti, N. Sambo, P. Castoldi, "On the Optimal Design of a Spectrum-Switched Optical Network With Multiple Modulation Formats and Rates", J. Opt. Commun. Netw., vol. 5, no. 11, pp. 1275-1284, 2013.

[14] Y. Sone, A. Hirano, A. Kadohata, M. Jinno, O. Ishida, "Routing and Spectrum Assignment Algorithm Maximizes Spectrum Utilization in Optical Networks," in Proc. ECOC 2011, Mo.1.K.3.

[15] S.P. Meyn, R. L. Tweedie, "Markov chains and stochastic stability”, Cambridge University Press, 2009. 
[16] J. Comellas, M. Ruiz, G. Junyent, " Optimizing Link Spectrum Occupancy in Elastic Optical Networks", to be presented at IEEE HPSR 2015.

[17] P. S. Khodashenas, D. Pomares, J. Perelló, S. Spadaro, J. Comellas, "A Comparison of Elastic and Multi-Rate Optical Networks Performance", in Proc. ICTON 2014, Mo.C1.1.

[18] Z. Shen, H. Hasegawa, K. Sato, T. Tanaka, A. Hirano, "A novel elastic optical path network that utilizes bitrate-specific anchored frequency slot arrangement", Optics Express, Vol. 22, Issue 3, pp. 3169-3179, 2014.

[19] K. Christodoulopoulos, I. Tomkos, E. A. Varvarigos, "Routing and Spectrum Allocation in OFDM-based Optical Networks with Elastic Bandwidth Allocation", in Proc. IEEE Globecom 2010, ONS06.

[20] Y. Wang, X. Cao, Y. Pan, "A Study of the Routing and Spectrum Allocation in Spectrum-sliced Elastic Optical Path Networks", in Proc. INFOCOM 2011, pp. 1503-1511..

[21] K. Christodoulopoulos, I. Tomkos, E. Varvarigos, "Elastic Bandwidth Allocation in Flexible OFDM-based Optical Networks," J. Lightw. Technol., vol. 29, no. 9, pp. 1354-1366, 2011.

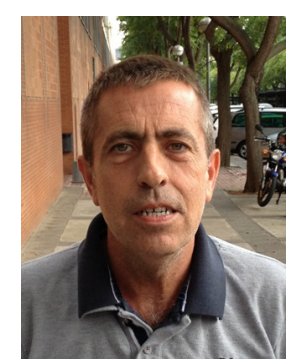

Jaume Comellas received the M.S. (1993) and Ph.D. (1999) degrees in Electrical Engineering from Universitat Politècnica de Catalunya (UPC), Spain. His current research interests focus on IP over Optical networking topics. He has participated in many research projects funded by the Spanish government as well as the European Commission. He has co-authored more than 100 research articles in international journals and conferences. $\mathrm{He}$ is an associate professor at the Signal Theory and Communications Department of UPC.

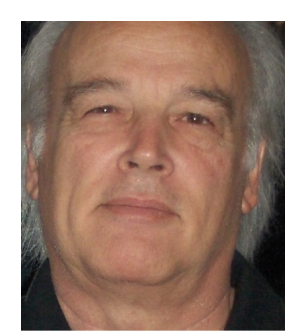

Gabriel Junyent graduated in telecommunication engineering from the Universidad Politécnica de Madrid, Spain, in 1973 , and received the Ph.D. degree in communications from the Universitat Politècnica de Catalunya (UPC), Barcelona, Spain, in 1979. From 1973 to 1989 , he was a Teaching Assistant and Associate Professor at UPC, where he has been a Full Professor since 1989. He founded the Advanced Broadband Communications Center (CCABA) at UPC in 1990. In the last 15 years he has participated in more than 30 national and international R\&D projects. $\mathrm{He}$ is coauthor of more than 150 journal and conference papers. 